\title{
Observations of interplanetary scintillation during the 1998 Whole Sun Month: a comparison between EISCAT, ORT and Nagoya data
}

\author{
P. J. Moran ${ }^{1}$, S. Ananthakrishnan ${ }^{2}$, V. Balasubramanian ${ }^{3}$, A. R. Breen ${ }^{1}$, A. Canals ${ }^{1}$, \\ R. A. Fallows ${ }^{1}$, P. Janardhan ${ }^{4,}$, M. Tokumaru ${ }^{5}$, P. J. S. Williams ${ }^{1}$ \\ ${ }^{1}$ Physics Department, University of Wales Aberystwyth, Penglais Hill, Aberystwyth, SY23 3BZ, UK \\ ${ }^{2}$ National Centre for Radio Astrophysics, TIFR, PO Box 3, Ganeshkhind, Pune 411 007, India \\ ${ }^{3}$ Radio Astronomy Centre, TIFR, PO Box 8, Udhagamandalam 643 001, India \\ ${ }^{4}$ Department of Astronomy, University of Maryland, College Park, MD 20742, USA \\ ${ }^{5}$ Solar-Terrestrial Environment Laboratory, Nagoya University, Toyokawa, Aichi 442, Japan \\ * On leave from the Physical Research Laboratory, Ahmedabad 380 009, India
}

Received: 3 April 2000 / Revised: 30 June 2000 / Accepted: 4 July 2000

\begin{abstract}
Observations of interplanetary scintillation (IPS) allow accurate solar wind velocity measurements to be made at all heliographic latitudes and at a range of distances from the Sun. The data may be obtained with either single, double or multiple antennas, each requiring a different method of analysis. IPS data taken during the 1998 whole sun month (30th July-31st August 1998) by EISCAT, the ORT (Ooty Radio Telescope), India, and the Nagoya IPS system, Japan, allow the results of individual methods of analysis to be compared. Good agreement is found between the velocity measurements using each method, and when combined an improved understanding of the structure of the solar wind can be obtained.
\end{abstract}

Key words: Interplanetary physics (solar wind plasma; sources of the solar wind) - Solar physics, astrophysics and astronomy (instruments and techniques)

\section{Introduction}

Planar wave-fronts from compact radio sources (e.g. quasars) are distorted as they pass through the solar wind creating a moving diffraction pattern at the Earth. Observations of this shifting pattern, known as interplanetary scintillation (IPS), allow properties of the solar wind, such as velocity, flow direction and r.m.s. electron density fluctuations, to be determined. For over thirty years, IPS has been used to make measurements at all heliographic latitudes and at a wide range of distances, from inside 10 solar radii out to beyond Earth's orbit (Rickett and Coles, 1991; Coles, 1995).

The earliest multi-site observations were used to obtain values of scintillation index and solar wind

Correspondence to: P. J. Moran

e-mail: plm@aber.ac.uk velocity (e.g. Dennison and Hewish, 1967; Hewish and Symonds, 1969), and later dual-site experiments provided continuity and improvements in the analysis technique (e.g. Armstrong and Coles, 1972). The accuracy of velocity measurements was found to improve as the baseline between sites increased (Bourgois et al., 1985) and with some observations, this allowed more than one distinct velocity lying along the line-of-sight to be resolved (Grall et al., 1996).

In recent years, much work has been published on several aspects of the solar wind, such as:-

1. The acceleration region (Grall et al., 1996; Breen et al., 2000)

2. The large-scale structure of solar wind (Moran et al., 1997; Kojima et al., 1998; Asai et al., 1998; Breen et al., 1999)

3. Corotating interaction regions and travelling interplanetary disturbances (Breen et al., 1997; Janardhan et al., 1996; Manoharan et al., 1995; Tokumaru et al., 2000)

4. The large-scale flow direction of the high latitude wind (Moran et al., 1998)

IPS is now accepted as a powerful ground-based tool for probing the structure of the inner heliosphere, and several groups around the world regularly make a number of different types of observation. This work compares results from observations made by the Ooty Radio telescope (ORT, Selvanagayam et al., 1993), the European Incoherent Scatter Radar (EISCAT, Rishbeth and Williams, 1985) and the Nagoya array in Japan (Kojima and Kakinuma, 1990). The ORT is a single antenna operating at $327 \mathrm{MHz}$, EISCAT comprises three radio telescopes in Northern Scandinavia operating at $931 \mathrm{MHz}$ and the Nagoya array comprises four radio telescopes operating at $327 \mathrm{MHz}$.

The observing frequency places constraints on the distance from the Sun at which IPS is reliably measured and interpreted. All observations require the plasma density to produce sufficient scattering power and cannot, therefore, be made beyond an outer limit. For single-site observations, the inner limit is defined by the 
need to be in a region of weak scattering (Hewish, 1951). Closer to the Sun, in strong scattering, it is not possible to calculate the spatial scale of irregularities using a single station and velocity measurements can not be made. Multi-site observations, however, can make measurements closer in, although they are less accurate than measurements made in weak scattering.

The transition region between strong and weak scattering differs in regions of fast and slow solar wind. At solar minimum, the slow equatorial wind is denser than the fast polar wind so that observations must be made further out from the Sun. The transition region is found near the peak in scintillation index with solar distance and can be measured experimentally. The observing window for ORT is approximately 40 to 200 solar radii, whereas Nagoya can make some measurements in as far as 20 solar radii. At the higher frequency, EISCAT can make measurements in weak scattering between approximately 15 and 120 solar radii.

The ORT makes nearly 100 observations per day, whereas EISCAT requires the observation to be made when the antennas' lines-of-sight lie parallel to the radial plane passing through the centre of the Sun. The orientation of the three EISCAT telescopes with respect to the solar wind changes as the Earth rotates about its axis and in general there are no more than 10 observations per day for which both geometry and source characteristics are favourable.

The four Nagoya telescopes allow a greater number of opportunities when the geometry between two sites is favourable, and reliable velocity data are available from approximately 40 radio sources a day. However, the largest baseline between antennas is $207 \mathrm{~km}$, so that the ability to resolve more than one distinct velocity along the line-of-sight in a single observation is limited. EISCAT, however, has a maximum baseline of $350 \mathrm{~km}$ and in some circumstances the signatures of two distinct velocities can be seen in the cross-correlation function of the power spectra (Breen et al., 1996a). The Japanese group resolve velocities by combining data taken over several complete solar rotations to create synoptic maps of large scale heliospheric activity (Jackson et al., 1997; Asai et al., 1998; Kojima et al., 1998).

\section{Observations and analysis}

During the 1998 Whole Sun Month (WSM2, 30 July 1998-31 August, 1998), ORT, Nagoya and EISCAT made a total of 1369,824 and 45 observations respectively from which solar wind velocities could be determined.

Such large numbers of observations allow ORT and Nagoya to make measurements of the heliosphere with far greater spatial resolution than EISCAT, enabling them to track transient events such as coronal mass ejections (CMEs) over a period of days. Figure 1 shows example plots of solar wind velocity measurements from the three systems as a function of solar elongation (defined as the angle subtended between the Earth/Sun line and the Earth/radio source line).
The fast velocity values on the East limbs in Fig. 1a suggest a transient event moving outwards from the Sun. The event is not so clearly evident in the Nagoya data, Fig. 1b, owing to lower spatial resolution. However, analysis of the data to obtain scintillation index, which gives an indication of plasma turbulence, shows enhancements that are consistent with ORT. Corresponding effects were also measured by spacecraft and this work is reported in separate papers (Tokumaru et al., 2000; Ananthakrishnan et al., 1999). Figure 1c shows the equivalent EISCAT plots. These measurements are on the opposite limb to the proposed CME, but quite clearly, the spatial resolution would be too low to be able to determine the presence of any such event.

Velocity estimates from single site IPS observations made by ORT are obtained by modelling the power spectra (Manoharan and Ananthakrishnan, 1990). A similar approach is taken with the EISCAT and Nagoya data, although as each observation involves two telescopes, the cross-correlation function of the spectra may also be modelled.

The diffraction pattern observed at the Earth is caused by scattering along the whole line-of-sight. In weak scattering, the model sums the scattering power from each point along this line-of-sight, giving each contribution a weighting that is proportional to $\mathrm{R}^{-4}$, where $\mathrm{R}$ is distance from the Sun. The weighting assumes a uniformly expanding solar atmosphere and such a rapid drop-off with distance, therefore, means that the majority of scattering occurs in the region where $\mathrm{R}$ is at a minimum (i.e. the point of closest approach to the Sun). The model used for ORT and EISCAT data calculates scattering to values of $\mathrm{R}$ at which the contribution is no longer significant. The Nagoya data is modelled to a distance from the Earth of 2 AU. Again, scattering from beyond this point is negligible.

If the radial flux of plasma is relatively uniform, a condition often found near solar minimum for observations at high or low solar latitude, both single and dual-site methods can provide reliable velocity values. However, when there are two or more distinct velocities present, it is necessary to know the region along the line-of-sight occupied by each flow type in order to determine their mean values. Two widely spaced antennas can sometimes resolve such velocities (Jackson et al., 1998; Breen et al., 1996b), but this is more difficult with a single antenna observation and any modelled velocity, therefore, will represent the weighted mean of the two velocities crossing the line-of-sight. The accuracy of velocity estimates also depends on factors such as signal to noise ratio and duration of the observation, and for both observation methods, the modelling is improved with extra information about the heliosphere.

ORT benefits from the large number of measurements so that the projection effects of observations that are both spatially and temporally close to each other, each of which will have different weighted values of velocity, may be used to model two stream velocities. EISCAT, having far fewer measurements, utilises white light coronagraphs taken by the High Altitude 
a) ORT

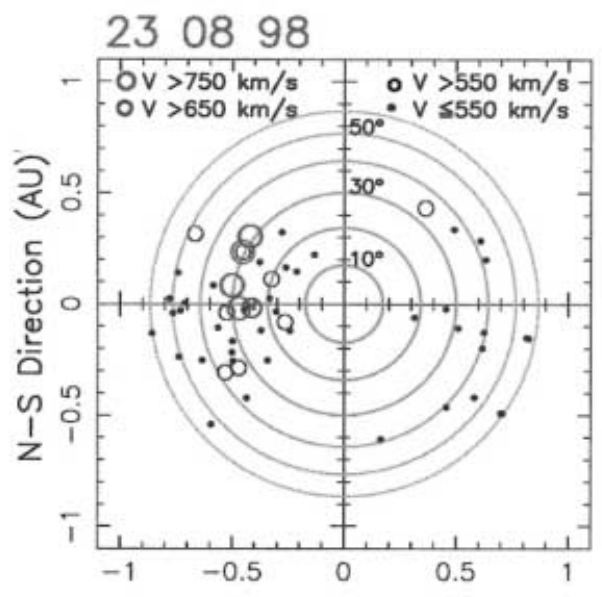

E-W Direction (AU)

b) Nagoya

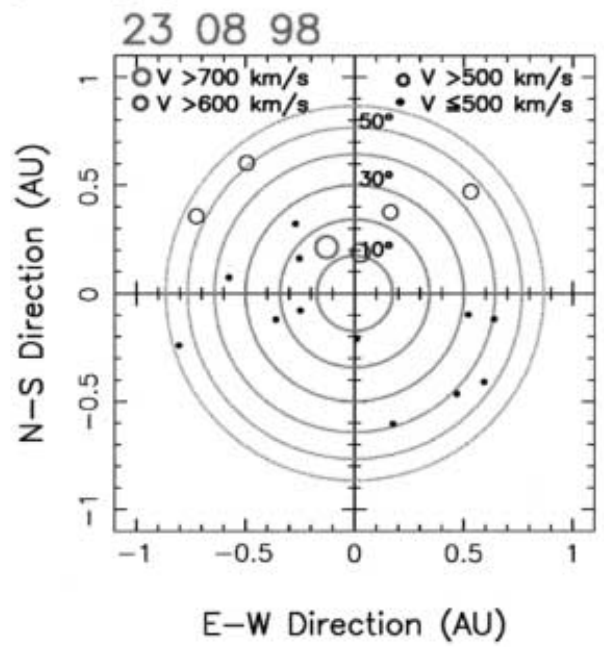

c) EISCAT

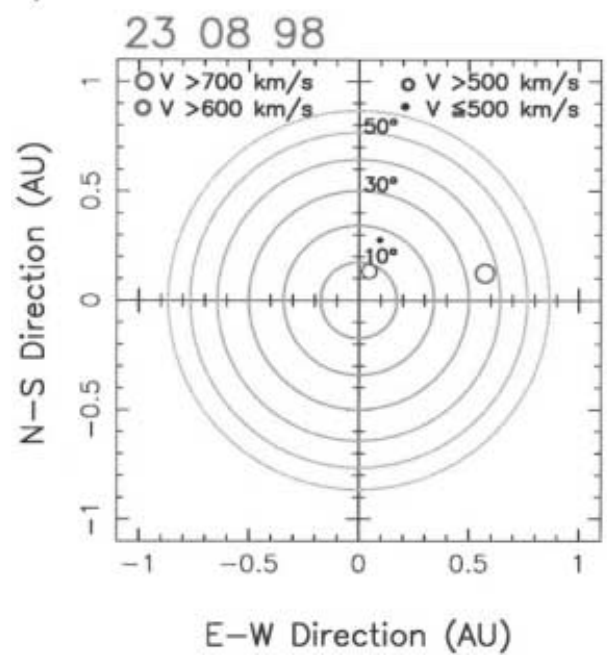

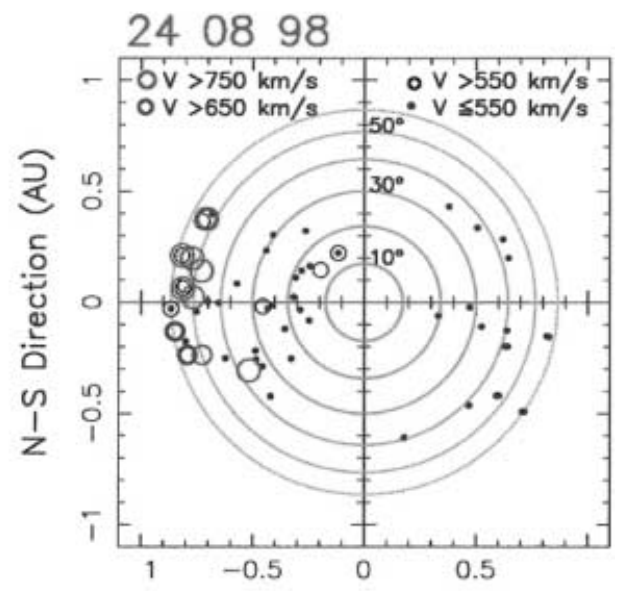

E-W Direction (AU)
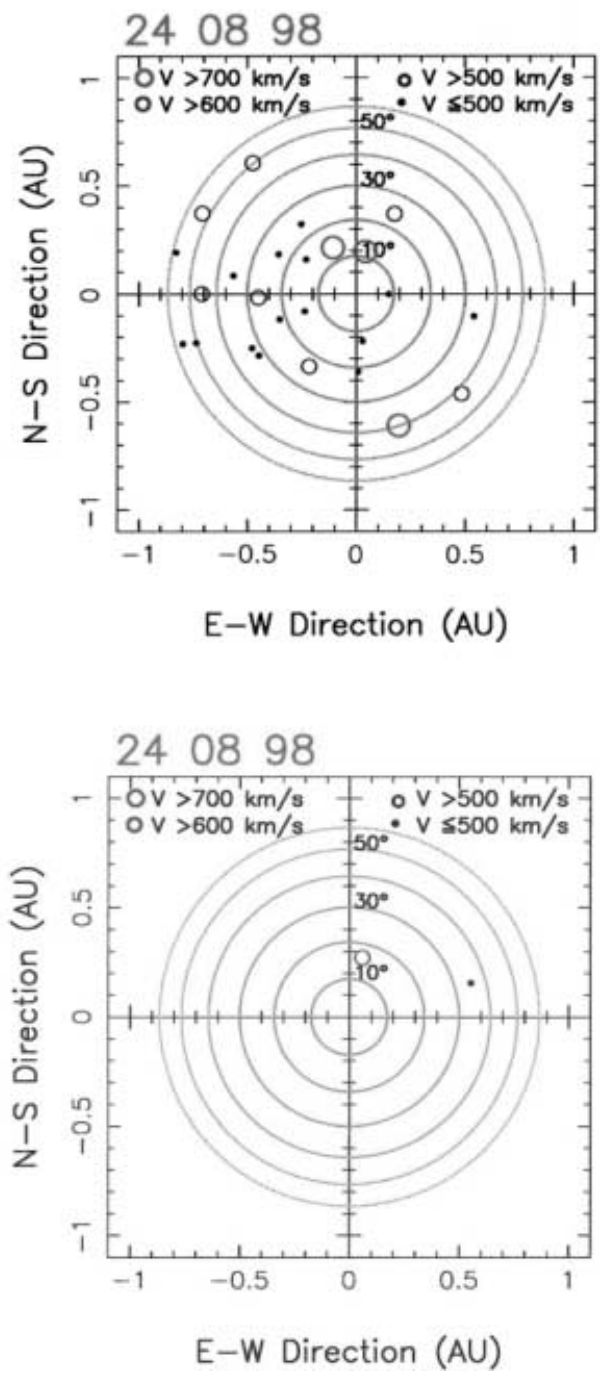

Fig. 1. Solar wind velocity as a function of solar elongation. The axes outside the plots are labelled in terms of astronomical units, whereas inside they are labelled in degrees. The circles of constant radial distance are included to help guide the eye
Observatory (HAO) to constrain the modelling. By mapping the radial flow crossing the line-of-sight back to the Sun's surface, the HAO maps allow an estimate to be made of the regions from which fast and slow flow emerges, enabling the two velocities to be modelled fairly easily (Breen et al., 1996a). 


\section{Results}

A specific comparison between the three data-sets may be made between observations of the same source for EISCAT and ORT, and mapping the lines-of-sight for these observations back to the Sun's surface as represented by the Nagoya synoptic maps. There were a total of four simultaneous observations made by EISCAT and ORT during the WSM2, the velocity estimates for which are shown in Table 1. There were no simultaneous observations with EISCAT and Nagoya because of their positions in different hemispheres of the Earth.

All velocity estimates match well, except for the observation made on 980814 in which the EISCAT data indicated two streams crossing the line-of-sight. The fitting method included estimates of the proportion of the line-of-sight lying in fast and slow flow and the relative amount of scattering per unit length between them. The 'primary' and 'secondary' velocities, therefore, indicate the dominance of the scattering power produced by each stream.

Figure 2 shows the lines-of-sight for each observation mapped back to the Nagoya synoptic map produced for the Whole Sun Month. It is more difficult to make a direct comparison of velocities between individual IPS measurements and the synoptic map seeing as the map shows regions of flow that are stable over a complete solar rotation. Nevertheless, it is clear that the complete line-of-sight for all the observations of the strongly scintillating quasar 3C273 (1229 + 020) made between the 23 to 25 August lie below the $550 \mathrm{~km} / \mathrm{s}$ contour. They also tend to lie parallel to the contour lines, especially at the point of closest approach. The minimum and maximum values indicated by the contour lines
Table 1. Velocity estimates made by EISCAT and ORT (see text)

\begin{tabular}{|c|c|c|c|c|c|c|}
\hline \multirow[t]{3}{*}{ Date } & \multirow[t]{3}{*}{ Source } & \multirow{3}{*}{$\begin{array}{l}\text { Helio latitude } \\
\text { (degree) }\end{array}$} & \multirow{3}{*}{$\begin{array}{l}\text { Radial distance } \\
(\mathrm{R} \odot)\end{array}$} & \multicolumn{3}{|c|}{ Velocity (km/s) } \\
\hline & & & & \multicolumn{2}{|l|}{ EISCAT } & \multirow[t]{2}{*}{ ORT } \\
\hline & & & & Primary & Secondary & \\
\hline 980814 & $1120+143$ & 20.2 & 92 & $820 \pm 80$ & $411 \pm 29$ & $500 \pm 25$ \\
\hline 980823 & $1229+020$ & 9.1 & 126 & $487 \pm 21$ & & $475 \pm 25$ \\
\hline 980824 & $1229+020$ & 9.3 & 124 & $369 \pm 20$ & & $300 \pm 50$ \\
\hline 980825 & $1229+020$ & 9.5 & 121 & $529 \pm 16$ & & $550 \pm 50$ \\
\hline
\end{tabular}

\section{SOLAR WIND SYNOPTIC MAP. CARRINGTON ROTATION 1939}

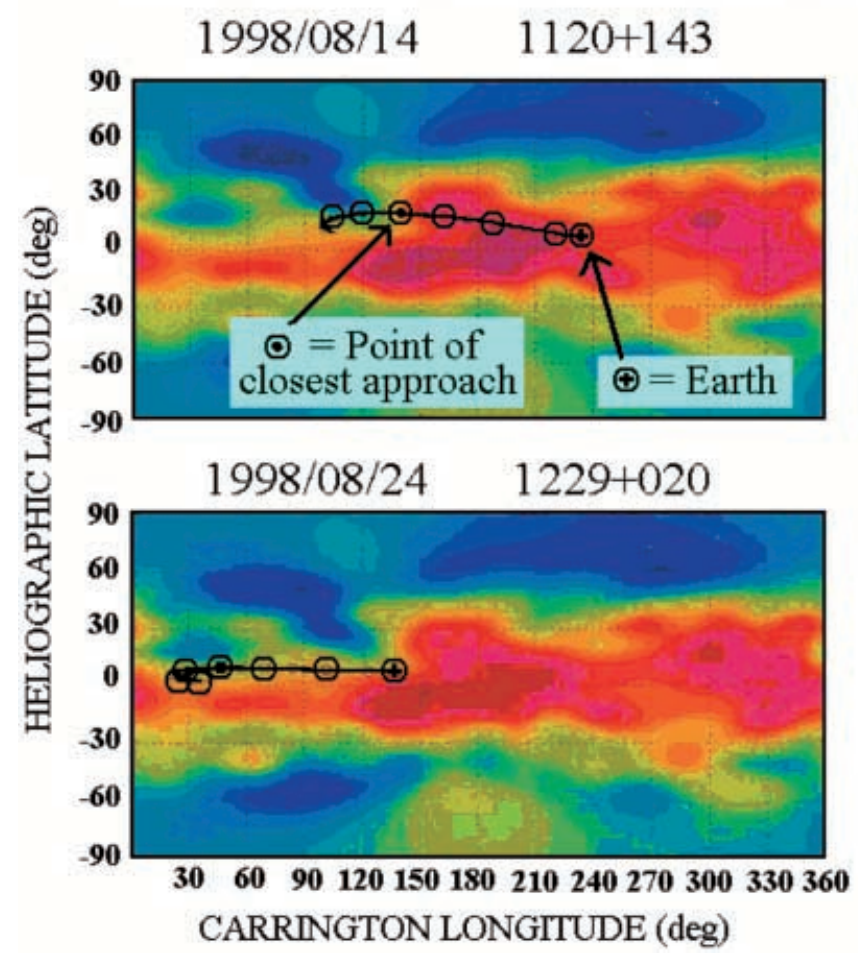

Fig. 2. Lines-of-sight for the simultaneous ORT/EISCAT observations mapped back to the synoptic map constructed from the Nagoya IPS data. The lines-of-sight are indicated by the solid black lines. The circled cross indicates where the line-of-sight reaches the Earth and the

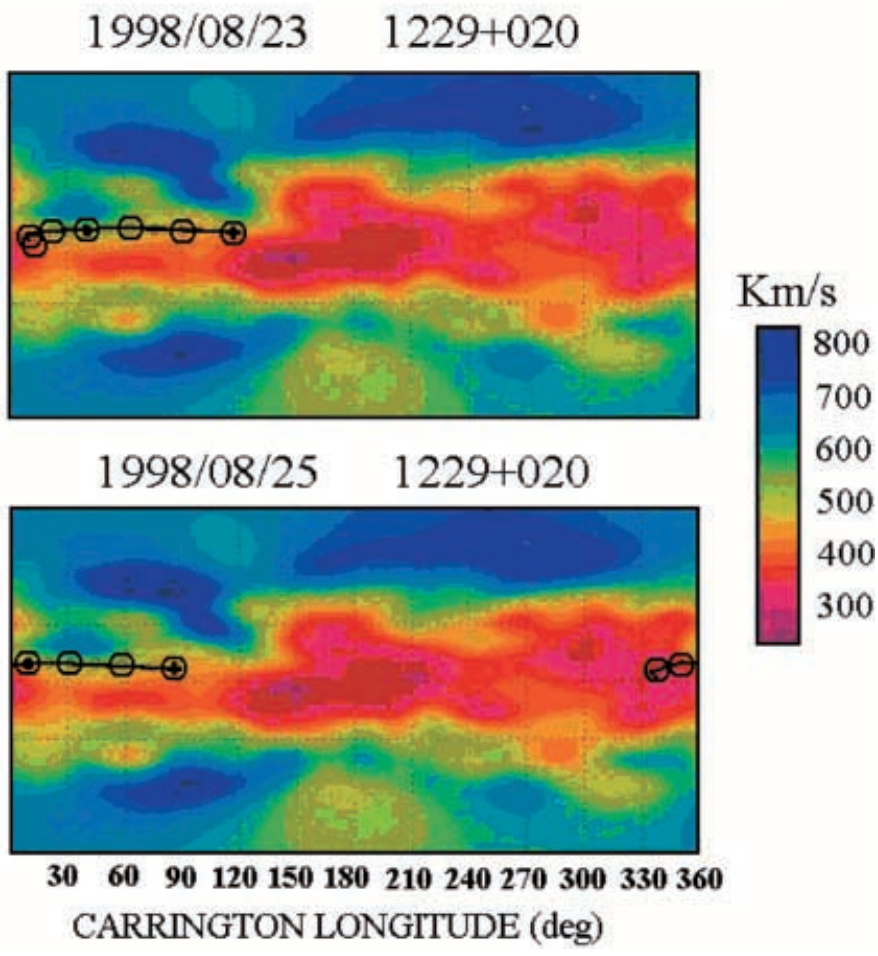

circled dot represents the point of closest approach to the Sun. Any point, P, along the line-of-sight may be considered in terms of an angle between $\mathrm{OP}$ and $\mathrm{O} \odot$, where $\mathrm{O}$ represents the centre of the Sun. Divisions of 20 degrees from $\mathbf{a r e}$ marked with circles 
are $425-500 \mathrm{~km} / \mathrm{s}, 375-500 \mathrm{~km} / \mathrm{s}$ and $300-525 \mathrm{~km} / \mathrm{s}$ on 23, 24 and 25 August respectively. Whilst all three sets of data on 23 August compare well, the EISCAT and ORT values are below those suggested by Nagoya for 24 August and above those suggested by Nagoya for 25 August. This suggests that ORT and EISCAT are picking up contributions of power from relatively shortterm features in the solar wind that are not detectable in the synoptic maps (although individual Nagoya observations would be able to pick up these features).

The observation on 14 August shows the line-of-sight to be orthogonal to the contours at the point of closest approach, giving weight to the validity of the EISCAT two stream velocity figures. The angle from the Earth to the point of closest approach is bounded by the 300 and $425 \mathrm{~km} / \mathrm{s}$ contour lines. Beyond this point, there is a sharp gradient between 0 and $10^{\circ}$ in which the velocity rises from 425 to $550 \mathrm{~km} / \mathrm{s}$. At approximately $35^{\circ}$ beyond the point of closest approach, the line-of-sight enters slow flow again. The figures for slow flow agree well with the EISCAT value of $411 \mathrm{~km} / \mathrm{s}$. However, EISCAT suggests a fast stream of $820 \mathrm{~km} / \mathrm{s}$ that does not concur with the synoptic map. Again this suggests that this fast flow is a short-term feature, not present for a complete solar rotation.

The ORT observation for 14 August was reanalysed using a two-stream model. Modelling of the power spectra relies primarily on the position of the 'Fresnel knee', the point at which there is a sharp gradient change in the spectra. The signal to noise ratio was high for $1120+143$, and as the EISCAT result had shown, the scattering power from the fast stream was less dominant than the slow stream. Two Fresnel knees were identified in the power spectra of this ORT observation, which was consistent with the EISCAT result.
Figure 3 shows the raw spectra with four examples of a single stream fitted model. Figure 3 a shows a model velocity of $200 \mathrm{~km} / \mathrm{s}$; clearly inaccurate, but included for demonstration purposes. Figure $3 b$ shows the original model in which the velocity estimate is $500 \mathrm{~km} / \mathrm{s}$. Figure $3 \mathrm{c}$ and $\mathrm{d}$ show models where the two 'knees' in the spectra are fitted separately.

Quite clearly, these two fitted velocities of $425 \pm 25$ and $825 \pm 25 \mathrm{~km} / \mathrm{s}$ match the EISCAT fitted velocities of $411 \pm 29$ and $820 \pm 80 \mathrm{~km} / \mathrm{s}$ very closely.

\section{Conclusions}

Single, double and multiple antenna IPS observations make measurements of the solar wind that differ in spatial and temporal accuracy. Single antennas are able to make regular measurements at all heliographic latitudes, allowing the spatial signatures of transient events such as CMEs to be observed.

Double antennas can give more accurate velocity estimates and, if the baseline between them is large enough, can resolve two distinct velocities along the lineof-sight. Far fewer useful observations may be made owing to the need for the appropriate geometrical conditions to be met, so the ability to map the spatial signatures of transients is limited.

In theory multiple antennas should give the best of both worlds. The greater the number of telescopes, the greater the number of two-antenna combinations there are, allowing a large number of accurate observations to be made. However, owing to the bimodal nature of the solar wind speed during solar minimum, it is also often necessary to resolve more than one velocity. If the distance between the sites is insufficient to be able to do

\section{$1408981120+143$}
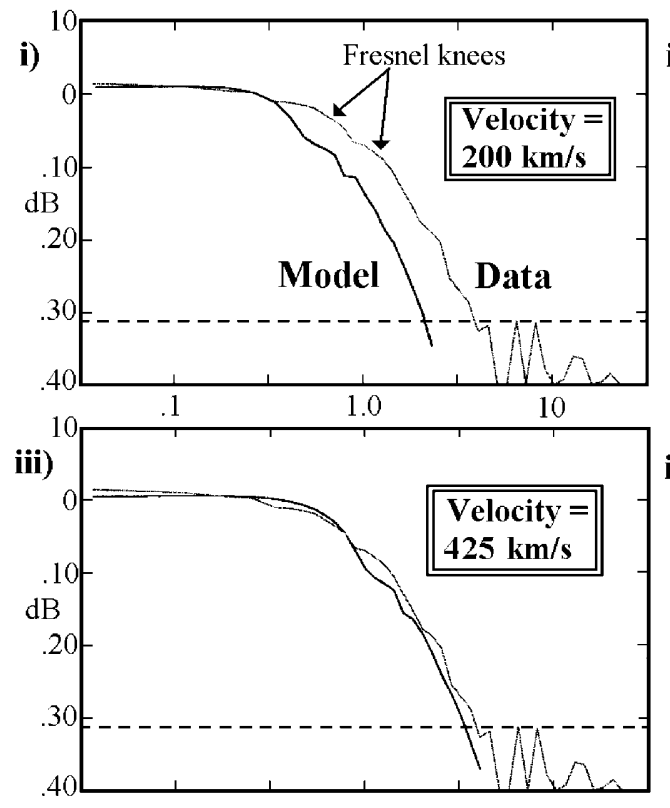

$\mathrm{Hz}$
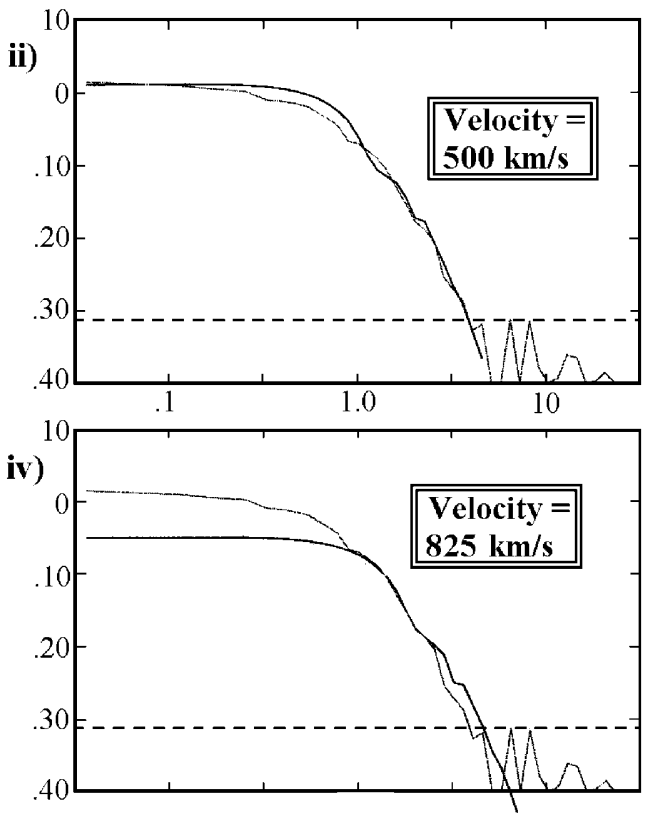

$\mathrm{Hz}$
Fig. 3a-d. Power spectra (thin line) of the observation of $1120+143$ on 14 August 1998 , with four examples of a fitted model (thick line). Clearly a modelled velocity of $200 \mathrm{~km} / \mathrm{s}$ does not match, as shown in $\mathbf{a}$. b Shows the best fit of the model if a single stream velocity is assumed. c, d Show the fits of the two Fresnel knees separately (indicated in a), each yielding a separate velocity. The dashed line indicates the power at which the spectrum falls to the noise level. In general, fits are only made when the signal to noise ratio of the spectra is greater than $20 \mathrm{~dB}$ 
this, a suitable alternative is to use tomographical analysis to build up a large-scale heliospheric map of solar wind activity. What such a map gains in spatial information it loses in temporal information, however, and it is not possible to detect solar events that occur for less than a few days using this method.

The three sets of IPS data from EISCAT, ORT and Nagoya reported in this study are shown to complement each other very well, allowing the accuracy of some individual observations to be enhanced.

Acknowledgement. Topical Editor M. Lester thanks W. Coles and E. Lucek for their help in evaluating this paper.

\section{References}

Ananthakrishnan, S., M. Kojima, M. Tokumaru, S. Balasubramanian, P. Janardhan, P. K. Manoharan, and M. Dryer, Study of solar wind transients using IPS, Proc. of Solar Wind 9 Conference, Eds. S. R. Habal, R. Esser, J. V. Hollweg, P. A. Isenberg, 471, pp 321-324, AIP Conference Proceessings, New York, 1999.

Armstrong, J. W., and W. A. Coles, Analysis of three-station interplanetary scintillation, J. Geophys. Res., 77, 4602-4610, 1972.

Asai, K., M. Kojima, M. Tokumaru, A. Yokobe, B. V. Jackson, P. L. Hick, and P. K. Manoharan, Heliospheric tomography using interplanetary scintillation observations 2 . Correlation between speed and electron density fluctuations in the solar wind, J. Geophys. Res., 103, 1991-2001, 1998.

Bourgois, G., W. A. Coles, G. Daigne, J. Silen, T. Turenen, and P. J. S. Williams, Measurements of the solar wind velocity with EISCAT, Astron. Astrophys., 144, 452-462, 1985.

Breen, A. R., W. A. Coles, R. Grall, U.P. Løvhaug, J. Markkanen, H. Misawa, and P. J. S. Williams, EISCAT measurements of interplanetary scintillation, J. Atmos. Terr. Phys., 58, 507-519, 1996a.

Breen, A. R., W. A. Coles, R. Grall, M. T. Klinglesmith, J. Markkanen, P. J. Moran, B. Tegid, and P. J. S. Williams, EISCAT measurements of the solar wind, Ann. Geophysicae, 14, 1235-1245, 1996b.

Breen, A. R., W. A. Coles, R. R. Grall, M. T. Klinglesmith, J. Markkanen, P. J. Moran, C. A. Varley, and P. J. S. Williams, EISCAT measurements of interaction regions in the solar wind, Adv. Space Res., 20, 27-30, 1997.

Breen, A. R., D. A. Biesecker, A. J. Lazarus, A. Lecinski, J. A. Linker, Z. Mikic, P. J. Moran, B. J. Thompson, C. A. Varley, and P. J. S. Williams, Interplanetary scintillation measurements of the solar wind during Whole Sun Month: comparisons with coronal and in situ observations, J. Geophys. Res. - Space Phys., 104(A5), 9847-9870, 1999.

Breen, A. R., C. F. DeForest, J. F. McKenzie, A. Modigliani, P. J. Moran, B. J. Thompson, C. A. Varley, and P. J. S. Williams, Comparisons of interplanetary scintillation and optical measurements of solar wind acceleration with model results, $A d v$. Space Res., 26(5), 781-784, 2000.
Coles, W. A., Interplanetary scintillation observations of the high latitude solar wind, Space Sci. Rev., 72(1/2), 211-222, 1995.

Dennison, P. A., and A. Hewish, The solar wind outside the plane of the ecliptic, Nature, 213, 343-346, 1967.

Grall, R. R., W. A. Coles, M. T. Klinglesmith, A. R. Breen, P. J. S. Williams, J. Markkanen, and R. Esser, Rapid acceleration of the polar solar wind, Nature, 379, 429-432, 1996.

Hewish, A., The diffraction of radio waves in passing through a phase-changing ionosphere, Proc. R. Soc. London, 209, 81-96, 1951.

Hewish, A., and M. D. Symonds, Radio investigation of the solar plasma, Planet Space Sci., 17, 313, 1969.

Jackson, B. V., P. L. Hick, M. Kojima, and A. Yokobe, Heliospheric tomography using interplanetary scintillation observations, Adv. Space Res., 20(1), 23-26, 1997.

Jackson, B. V., P. L. Hick, M. Kojima, and A. Yokobe, Heliospheric tomography using interplanetary scintillation observations: 1. Combined Nagoya and Cambridge data, J. Geophys. Res., 103, 12 049-12 067, 1998.

Janardhan, P., V. Balasubramanian, S. Ananthakrishnan, M. Dryer, A. Bhatnagar, and P. S. McIntosh, Travelling interplanetary disturbances detected using interplanetary scintillation at 327 MHz, Solar Phys., 166, 379-401, 1996.

Kojima, M., and T. Kakinuma, Solar-cycle dependence of global distribution of solar-wind speed, Space Sci. Rev., 53(3, 4), 173222, 1990.

Kojima, M., M. Tokumaru, H. Watanabe, A. Yokobe, K. Asai, B. V. Jackson, and P. L. Hick, Heliospheric tomography using interplanetary scintillation observations 2. Latitude and heliocentric distance dependence of solar wind structure at 0.1-1 AU, J. Geophys. Res., 103, 1981-1989, 1998.

Manoharan P. K., and S. Ananthakrishnan, Determination of solarwind velocities using single-station measurements of interplanetary scintillation. Mon. Notices R. Astron. Soc., 244(4), 691-695, 1990.

Manoharan P. K., S. Ananthakrishnan, M. Dryer, T. R. Detman, H. Leinbach, M. Kojima, T. Watanabe, and J. Khan, Solar-wind velocity and normalized scintillation index from single-station IPS observations. J. Sol. Phys., 156(2), 377-393, 1995.

Moran, P. J., A. R. Breen, W. A. Coles, R. Grall, M. T. Klinglesmith, J. Markkanen, C. A. Varley, and P. J. S. Williams, EISCAT measurements of the solar wind: measurements of fast and slow streams, Phys. Chem. Earth Sol. Sys., 22(5), 389-392, 1997.

Moran, P. J., A. R. Breen, J. Markkanen, C. A. Varley, W. P. Wilkinson, and P. J. S. Williams, Measurements of the direction of the Solar wind using interplanetary scintillation, Ann. Geophysicae, 16(10), 1259-1264, 1998.

Rickett, B. J., and W. A. Coles, Evolution of the solar wind structure over a solar cycle: interplanetary scintillation velocity measurements compared with coronal observations, J. Geophys. Res., 96(A2), 1717-1736, 1991.

Rishbeth, H., and P. J. S. Williams, Ionospheric radar: the system and its early results, R. Astron. Soc., 26, 478-512, 1985.

Selvanagayam, A. J., A. Praveenkumar, D. Nandagopal, and T. Veluswamy, IETE Technical Rev. 10(4), 333, 1993.

Tokumaru, M., M. Kojima, K. Fujiki, and A. Yokobe, Threedimensional propagation of interplanetary disturbances detected with radio scintillation measurements at $327 \mathrm{MHz}$, J. Geophys. Res., 105(A5), 10 435-10 453, 2000. 